\title{
Um panorama das publicações do Programa de Educação Tutorial relacionadas às atividades com o Ensino Médio
}

\author{
An overview of publications of the Tutorial Education Program related to High School activities \\ Resumen de las publicaciones del Programa de Educación Tutorial relacionadas con las actividades \\ de la Escuela Secundaria
}

Recebido: 20/09/2021 | Revisado: 26/09/2021 | Aceito: 28/09/2021 | Publicado: 01/10/2021

\author{
Andrielli da Silva Fontoura \\ ORCID: https://orcid.org/0000-0002-6245-154X \\ Universidade Federal de Santa Maria, Brasil \\ E-mail: andriellifontoura@gmail.com \\ Lenira Maria Nunes Sepel \\ ORCID: https://orcid.org/0000-0001-8372-057X \\ Universidade Federal de Santa Maria, Brasil \\ E-mail: lenirasepel@gmail.com
}

\begin{abstract}
Resumo
Objetivo: Mapear e compreender melhor os tipos de interações que existem entre as atividades desenvolvidas pelos grupos PET e o Ensino Médio. Metodologia: Esta é uma pesquisa bibliográfica, realizada a partir das buscas dos descritores "Programa de Educação Tutorial ou Educação Tutorial"; "Programa de Educação Tutorial e Ensino Médio" e "Educação Tutorial e Ensino Médio" nas bases de dados: Periódicos da Capes, Scielo, Google acadêmico e Biblioteca Digital Brasileira de Teses e Dissertações, publicados no período de janeiro de 2015 a março de 2020. Resultados e discussão: Foram analisadas 24 publicações. Onde foi possível identificar atividades multidisciplinares e disciplinares. Estas últimas estavam relacionadas à Biologia, Química, Física, Matemática, História e Português. Não foram identificadas atividades específicas nas disciplinas de Geografia, Artes, Educação Física, Sociologia, Filosofia e Inglês. Porém foi possível identificar algumas destas nas atividades multidisciplinares (Artes e Sociologia). As atividades ocorreram nas turmas de $1^{\circ}, 2^{\circ}$ e $3^{\circ}$ ano. Foram identificados diferentes tipos de atividades voltadas à leitura, interpretação, discussão e construção de textos; palestras e debates sobre diferentes temas relevantes; atividades práticas e oficinas nas diferentes áreas de estudo; construções, competições e concursos de materiais elaborados pelos alunos; apoio escolar; cinema; divulgação de cursos de graduação; entre outros cursos específicos ofertados. Considerações Finais: O modelo consolidado pelos grupos PET é uma possibilidade de adoção e parceria de atividades que tenham interação com o Ensino Médio, pensando na melhoria da qualidade do ensino básico, na continuidade dos estudos e na preparação profissional.
\end{abstract}

Palavras-chave: Educação tutorial; Ensino médio; Interações; Ensino.

\begin{abstract}
Objective: To map and better understand the types of interactions that exist between the activities developed by the PET groups and High School. Methodology: This is a bibliographical research, carried out from the searches of the descriptors "Tutorial Education Program or Tutorial Education"; "Tutorial Education and High School Program" and "Tutorial Education and High School" in the databases: Capes, Scielo, Academic Google and Brazilian Digital Library of Theses and Dissertations, published from January 2015 to March 2020 Results and discussion: 24 publications were analyzed. Where it was possible to identify multidisciplinary and disciplinary activities. The latter were related to Biology, Chemistry, Physics, Mathematics, History and Portuguese. No specific activities were identified in the subjects of Geography, Arts, Physical Education, Sociology, Philosophy and English. However, it was possible to identify some of these in multidisciplinary activities (Arts and Sociology). The activities took place in the 1st, 2nd and 3rd year classes. Different types of activities aimed at reading, interpreting, discussing and constructing texts were identified; lectures and debates on different relevant topics; practical activities and workshops in different areas of study; constructions, competitions and contests for materials designed by students; school support; movie theater; dissemination of undergraduate courses; among other specific courses offered. Final Considerations: The model consolidated by the PET groups is a possibility of adopting and partnering with activities that interact with High School, thinking about improving the quality of basic education, continuing studies and professional preparation.
\end{abstract}

Keywords: Tutorial education; High school; Interaction; Teaching. 


\begin{abstract}
Resumen
Objetivo: Mapear y comprender mejor los tipos de interacciones que existen entre las actividades desarrolladas por los grupos PET y Bachillerato. Metodología: Se trata de una investigación bibliográfica, realizada a partir de las búsquedas de los descriptores "Programa de Educación Tutorial o Educación Tutorial"; "Programa de Educación Tutorial y Bachillerato" y "Educación Tutorial y Bachillerato" en las bases de datos: Capes, Scielo, Google Académico y Biblioteca Digital Brasileña de Tesis y Disertaciones, publicados de enero de 2015 a marzo de 2020 Resultados y discusión: se analizaron 24 publicaciones. Donde fue posible identificar actividades multidisciplinarias y disciplinarias. Estos últimos estaban relacionados con Biología, Química, Física, Matemáticas, Historia y Portugués. No se identificaron actividades específicas en las asignaturas de Geografía, Artes, Educación Física, Sociología, Filosofía e Inglés. Sin embargo, fue posible identificar algunos de estos en actividades multidisciplinares (Artes y Sociología). Las actividades se desarrollaron en las clases de $1^{\circ}, 2^{\circ}$ y $3^{\circ}$ curso. Se identificaron diferentes tipos de actividades dirigidas a la lectura, interpretación, discusión y construcción de textos; conferencias y debates sobre diferentes temas relevantes; actividades prácticas y talleres en diferentes áreas de estudio; construcciones, concursos y concursos de materiales diseñados por estudiantes; apoyo escolar; cine; difusión de cursos de pregrado; entre otros cursos específicos ofrecidos. Consideraciones finales: El modelo consolidado por los grupos PET es la posibilidad de adoptar y asociar actividades que interactúen con Bachillerato, pensando en mejorar la calidad de la educación básica, los estudios continuos y la preparación profesional.
\end{abstract}

Palabras clave: Educación tutorial; Escuela secundaria; Interacción; Enseñanza.

\title{
1. Introdução
}

O Programa de Educação Tutorial (PET) foi criado em 1979 e atualmente é vinculado à Secretaria de Educação Superior do Ministério da Educação (SESu/ MEC); é desenvolvido por grupos de estudantes em nível de graduação, com tutoria de um docente. Os planejamentos dos grupos PET de todas as Instituições de Ensino Superior do País são orientados pelo princípio da indissociabilidade entre ensino, pesquisa e extensão e seguem o modelo da educação tutorial (Brasil, 2006).

O Manual de Orientações Básica do PET (Brasil, 2006) apresenta os objetivos do programa que incluem a promoção da formação acadêmica ampla e de qualidade para alunos de graduação envolvidos direta e indiretamente com o programa. Esses objetivos se manifestam no desenvolvimento de atividades variadas que estimulam e reforçam ações de cidadania e desenvolvimento de consciência social em todos os participantes, colaborando com a melhoria dos cursos de graduação.

O PET organiza e executa diferentes grupos de atividades que podem ser agrupadas em grandes associações: atividades com caráter principal de iniciação à pesquisa, atividades de complementação do ensino superior, visando à formação dos acadêmicos em áreas específicas do conhecimento e atividades de extensão que promovem interação com a comunidade na qual a instituição de ensino superior está inserida.

Desde que foi criado, o PET tem sido objeto de estudo, seja para caracterizar os impactos do Programa na formação acadêmica, seja para compreender melhor e aprimorar as dinâmicas de funcionamento desse modelo de educação tutorial. Um exemplo é a análise feita por Souza e Gomes Junior (2015) em relação ao PET Física da Universidade Federal do Rio Grande do Norte (UFRN) que foi o primeiro grupo PET na área de Física do país. Nesse trabalho, os autores apresentam uma análise do impacto do grupo PET no desempenho discente durante o curso de graduação. Os autores destacam entre os resultados as diferenças no tempo de graduação e na evasão de curso entre petianos (denominação dada aos alunos bolsistas ou voluntários do programa) e não petianos. Além disso, através do mapeamento da colocação profissional dos egressos do programa, Souza e Gomes Júnior (2015) analisaram a contribuição do PET para o ensino de Física na Educação Básica do RN. Na questão do tempo de graduação foi possível identificar que os petianos acabam o curso em menor tempo do que os não petianos; a evasão é maior entre os não petianos; e os ex-petianos são considerados mais bem sucedidos pela inserção no mercado de trabalho e em cursos de pós-graduação.

Dentre as atividades realizadas pelo grupo PET- Física/ UFRN, Souza e Gomes Júnior (2015) destacam: curso de nivelamento ministrado para estudantes ingressantes no curso de física; monitoria voluntária das disciplinas básicas do curso; realização de seminários semanais; participação em eventos; iniciação científica voluntária com professores doutores do 
Departamento de Física Teórica e Experimental (DFTE) - colaboradores do PET; realização de oficinas para o curso de licenciatura em Física da modalidade à distância da UFRN; abertura dos laboratórios de Física para visitação de estudantes do Ensino Fundamental e Médio.

Outros relatos e análises de atividades desenvolvidas por grupos PET relacionados às Ciências da Natureza são encontrados, por exemplo, nos trabalhos de Marmitt, Hermel e Friedrich (2016) e Bremm (2019). Em comum, esses relatos apresentam atividades de interação universidade - escola, dando destaque para a importância das atividades práticas para a aprendizagem de Ciências. Essas publicações também valorizam a contextualização de conhecimentos, buscando aproximar as informações científicas e situações do cotidiano dos estudantes, trabalhando conteúdos como as informações sobre educação alimentar.

Os grupos PET, embora desenvolvam ações pertinentes às áreas nas quais os graduandos estão obtendo formação, apresentam uma forte semelhança no desenvolvimento de atividades integradas, associando ensino, pesquisa e extensão. Semelhantes aos Programas dos grupos da área de Ciências da Natureza, os grupos PET de cursos das Ciências Humanas também atuam em interações com escolas de Educação Básica, com os objetivos de melhoria do ensino e de auxiliar estudantes do Ensino Médio a ingressar e adaptar-se ao Ensino Superior (Martins, Espíndola, Olanda \& Nunes, 2018; Silva, et al., 2017).

Desde o ano de 2017, as mudanças necessárias na Educação Básica, associadas às aprovações da Base Nacional Comum Curricular (BNCC) (Brasil, 2018) e da reforma do Ensino Médio (Brasil, 2017), têm sido objeto de muita discussão crítica. Reflexões sobre a capacidade das alterações curriculares prevista nesses documentos serem capazes de modificar o cenário de "excessiva disciplinarização e baseado no acúmulo de informações" (Silva, 2018, p.12) aparecem em várias publicações (Ferretti, 2018; Fernandes, 2019; \& Corti, 2019) que fazem uma leitura crítica e reflexiva sobre essas mudanças, que "não afetam apenas o ensino médio, mas também a vida e a formação dos professores, o exercício do magistério e as condições de autonomia didático-pedagógica das escolas" (Ciavatta, 2018, p. 209).

O Novo Ensino Médio é um novo modelo de ensino estabelecido pela reforma na Lei de Diretrizes e Bases da Educação Nacional, que altera a grade curricular aumentando o tempo mínimo do estudante na escola, possui uma organização curricular mais flexível e trabalha a partir de um currículo comum estabelecido pela BNCC. Além disso, também são ofertados itinerários formativos, focados em diferentes áreas de conhecimento, formação técnica e profissional; os quais têm como principal proposta aprofundar conhecimentos em áreas de maior aptidão e interesse. O MEC alterou a carga horária para reduzir a evasão escolar e melhorar a qualidade da educação que chega aos alunos. A medida é parte do Compromisso Nacional pela Educação Básica que pretende tornar o Brasil referência em educação básica na América Latina até 2030 (Brasil, 2019).

O modelo de educação tutorial, estruturado como o que é praticado nos grupos PET, pode ser adaptado e aplicado como auxiliar na implementação do Novo Ensino Médio. Ações que permitam a cada estudante aprofundar conhecimentos em áreas de maior aptidão e interesse não são uma tradição dos currículos escolares e demandam a inclusão de novas metodologias de trabalho para o processo de ensino-aprendizagem (Piffero, Soares, Coelho \& Roehrs, 2020).

Os efeitos da educação tutorial no ensino superior seja na redução da evasão, no engajamento e motivação para a progressão nos estudos ou na formação de lideranças positivas (Silva, Bassani, \& Santos, 2017) são amplamente reconhecidos. A possibilidade de desenvolver, para o contexto do Novo Ensino Médio, atividades que sejam amparadas pela experiência do PET é uma proposta a ser desenvolvida. Nesse sentido, o presente trabalho tem como objetivo mapear e compreender melhor os tipos de interações que existem entre as atividades desenvolvidas pelos grupos PET e o Ensino Médio. A pesquisa teve como base as publicações dos grupos PET que foram analisadas e categorizadas de acordo com as disciplinas e o tipo de ação. 


\section{Metodologia}

Conforme delineamento estabelecido por Gil (2018) esta pesquisa foi classificada como pesquisa bibliográfica, a qual é elaborada com base em material já publicado e obtido em bases de dados. Pereira, et al. (2018) considera que nessa metodologia "um autor realiza a busca, leitura, análise: discute os resultados obtidos em relação aos autores consultados nas referências e, escreve uma conclusão ou várias conclusões em relação a um problema da pesquisa ou assunto".

O desenvolvimento da pesquisa foi dividido nas seguintes etapas: $i$ ) definição do tema e dos descritores; ii) seleção das bases de dados para busca; iii) determinação dos critérios de inclusão e exclusão de publicações; iv) análise dos resultados das buscas e discussão.

A pesquisa foi destinada a coletar relatos de atividades de extensão realizadas pelos Grupos PET que tivessem relação com o Ensino Médio. Quatro descritores foram selecionados, dois gerais e duas associações específicas para o objetivo do trabalho. Para encontrar um conjunto mais diversificado de publicações foram utilizados os termos Programa de Educação Tutorial ou Educação Tutorial. As associações "Programa de Educação Tutorial e Ensino Médio" e "Educação Tutorial e Ensino Médio" foram empregadas para encontrar relatos de interação dos grupos PET com a Educação Básica. É importante ressaltar que optamos por não pesquisar a palavra "PET", pois quando utilizada a sigla para busca os principais resultados estão relacionados ao "poli tereftalato de etila", que é um tipo de polímero plástico, ou seja, a conhecida garrafa PET. Ou ainda, pode ser encontrado como termo em inglês utilizado para animais de estimação.

O período de realização das buscas de publicações foi de fevereiro a março de 2020. O acesso foi através das bases de dados dos Periódicos da Capes, Scielo, Google acadêmico e Biblioteca Digital Brasileira de Teses e Dissertações (BDTD).

$\mathrm{Na}$ etapa de inclusão e exclusão de publicações para análise, foram aplicados dois critérios: publicações ocorridas de janeiro de 2015 a março de 2020, independente do período de realização das atividades relatadas; presença de relatos com qualquer tipo de atividade de ensino, independentemente do nível no qual foi aplicada. Para os termos mais amplos, pesquisados no Google Acadêmico foi previamente estabelecido que apenas a primeira centena de resultados passaria pela triagem inicial.

Após a triagem inicial, foi realizada a leitura dos resumos dos trabalhos coletados e excluídos aqueles que apareceram com duplicidade ou não possuíam links ativos ou não remetiam a publicações completas.

As publicações, cujos resumos foram selecionados na etapa de leitura inicial, constituíram uma segunda amostra para análise de categorias e essa amostra foi utilizada para descrever as atividades dos grupos PET com o Ensino Médio.

\section{Resultados e Discussão}

No total, para as quatro bases de dados pesquisadas, foram encontradas 7.925 publicações referentes a atividades de grupos PET. Na base de dados Periódicos da Capes foram encontrados 52 resultados; no Scielo identificou-se 7 publicações e a Base Digital Brasileira de Teses e Dissertações teve um total de 6 produções relacionadas com atividades de grupos PET e ensino. De acordo com o previsto, o resultado da busca na base de dados do Google acadêmico foi muito maior (7.860 itens) quando as expressões de busca empregadas foram Programa de Educação Tutorial ou Educação Tutorial. O critério prévio de leitura inicial da primeira centena de resultados do Google acadêmico foi aplicado e resultou em 75 publicações sem repetições e com links válidos para trabalhos completos (resumos excluídos). Após essa triagem as publicações resultaram em 140 trabalhos das quatro bases de dados.

A leitura completa dessas publicações foi realizada e resultou em um novo conjunto de exclusões. Foram removidos trabalhos duplicados e/ou com relatos incompletos (sem especificação de ações desenvolvidas) ou sem vínculo direto com a Educação Básica. No final do processo de seleção de trabalhos analisáveis, com relatos específicos de atividades em escolas, 
obteve-se uma amostra constituída por 56 publicações, e relacionadas somente ao Ensino Médio foram 24 publicações. Abaixo o Quadro 1 traz os resultados do universo da pesquisa.

Quadro 1- Arquivos selecionados sobre os descritores nas diferentes bases de dados.

\begin{tabular}{|c|c|c|c|c|c|}
\hline Base de dados & Periódicos Capes & Scielo & Google Acadêmico & BDTD & TOTAL \\
Descritores & & 17 & 10 & 4 & 32 \\
\hline Programa de Educação Tutorial & 0 & 0 & 21 & 21 \\
\hline Programa de Educação Tutorial e Ensino \\
Médio
\end{tabular}

Fonte: Autores.

Como é possível verificar no Quadro 1, a maioria das 56 publicações selecionadas está associada ao descritor Programa de Educação Tutorial e foi encontrada no portal de Periódicos da Capes. Para a combinação Programa de Educação Tutorial e Ensino Médio e para a combinação Educação Tutorial e Ensino Médio foram encontrados respectivamente 21 e 3 publicações no Google Acadêmico.

É importante ressaltar que não foram selecionadas publicações referentes ao descritor Educação Tutorial, pois todas as obtidas com essa expressão também apareceram com o descritor Programa de Educação Tutorial e foram consideradas duplicações.

Gráfico 1- Comparação do número de publicações por ano nas diferentes bases de dados.

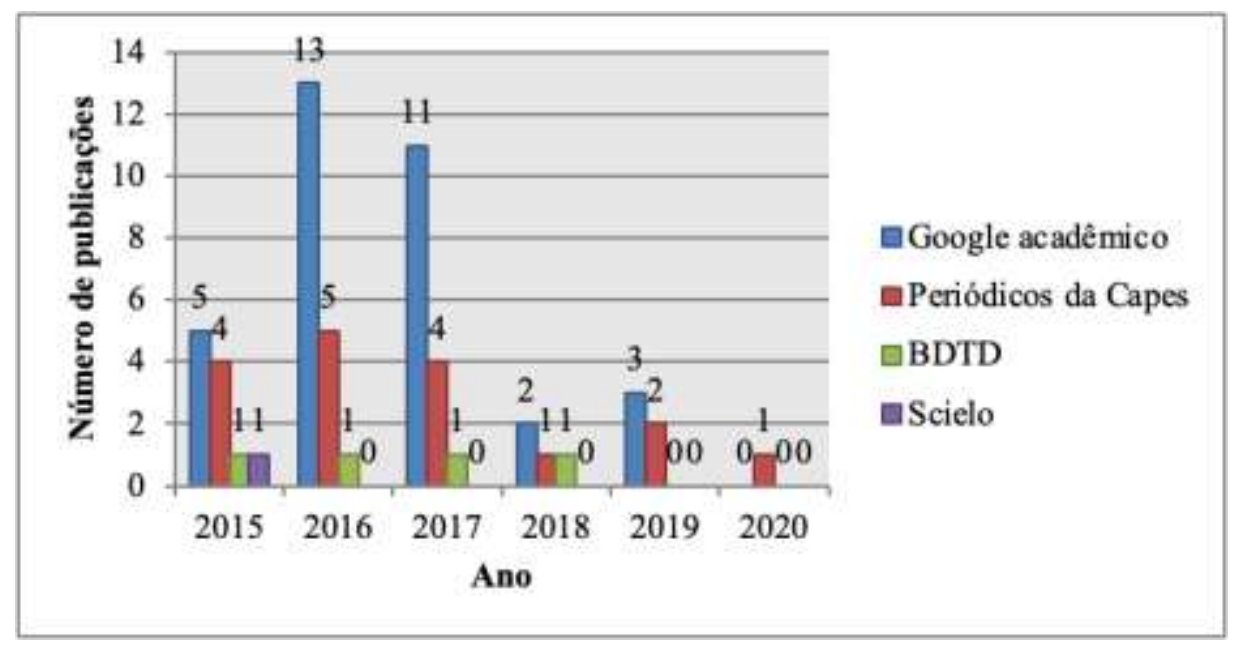

Fonte: Autores.

Como se verifica no Gráfico 1, a maior produção dos Grupos PET ocorreu do ano de 2015 à 2017, principalmente com publicações disponíveis no Google acadêmico. Esse resultado reflete o fato do sistema Google Acadêmico incluir uma 
grande variedade de tipos de publicações, especialmente as que são produzidas em eventos acadêmicos nas áreas de ensino, pesquisa e extensão. Diferente das outras bases de dados que são constituídas por publicações mais especializadas sob a forma de artigos científicos, livros, dissertações e teses, por exemplo.

As publicações do Google Acadêmico incluem resumos, resumos expandidos e trabalhos completos publicados em congressos, simpósios, seminários e encontros universitários. Essas modalidades de eventos são consideradas muito relevantes para a formação dos petianos que usualmente têm incentivos institucionais para participação. O trabalho realizado por Dearo, Nakayama e Rossit (2017) com objetivo conhecer a percepção dos acadêmicos com relação às possíveis potencialidades e fragilidades encontradas no PET das diferentes regiões do Brasil, se refere à essa situação. Foi identificado no relato de participantes da pesquisa "dificuldade no que tange ao financiamento de congressos, pesquisas, materiais de uso permanente" e a "desvalorização como programa, em nível governamental e institucional" (Dearo, et al., 2017). Esses resultados indicam problemas na destinação dos recursos que amparam o funcionamento dos grupos PET e vão de encontro à realidade dos petianos, que dependem das bolsas e custeio para a participação em eventos.

A análise do número de publicações dos grupos PET apresenta uma redução a partir do ano de 2018; pode-se perceber a queda no número de publicações associadas aos grupos PET nas quatro bases de dados. Chegando em 2020, considerado um ano atípico devido ao início da pandemia do coronavírus, com a suspensão de todos os eventos presenciais previstos para o ano, pode-se supor que o número de publicações terá uma redução extrema. O impacto que terão os eventos cancelados e as atividades presenciais no ensino se tornando remotas ainda deve ser registrado e analisado, pois foi um ano de adaptação para todos.

Figura 1- Resultados da classificação das atividades desenvolvidas pelos grupos PET com interação com o Ensino Médio.

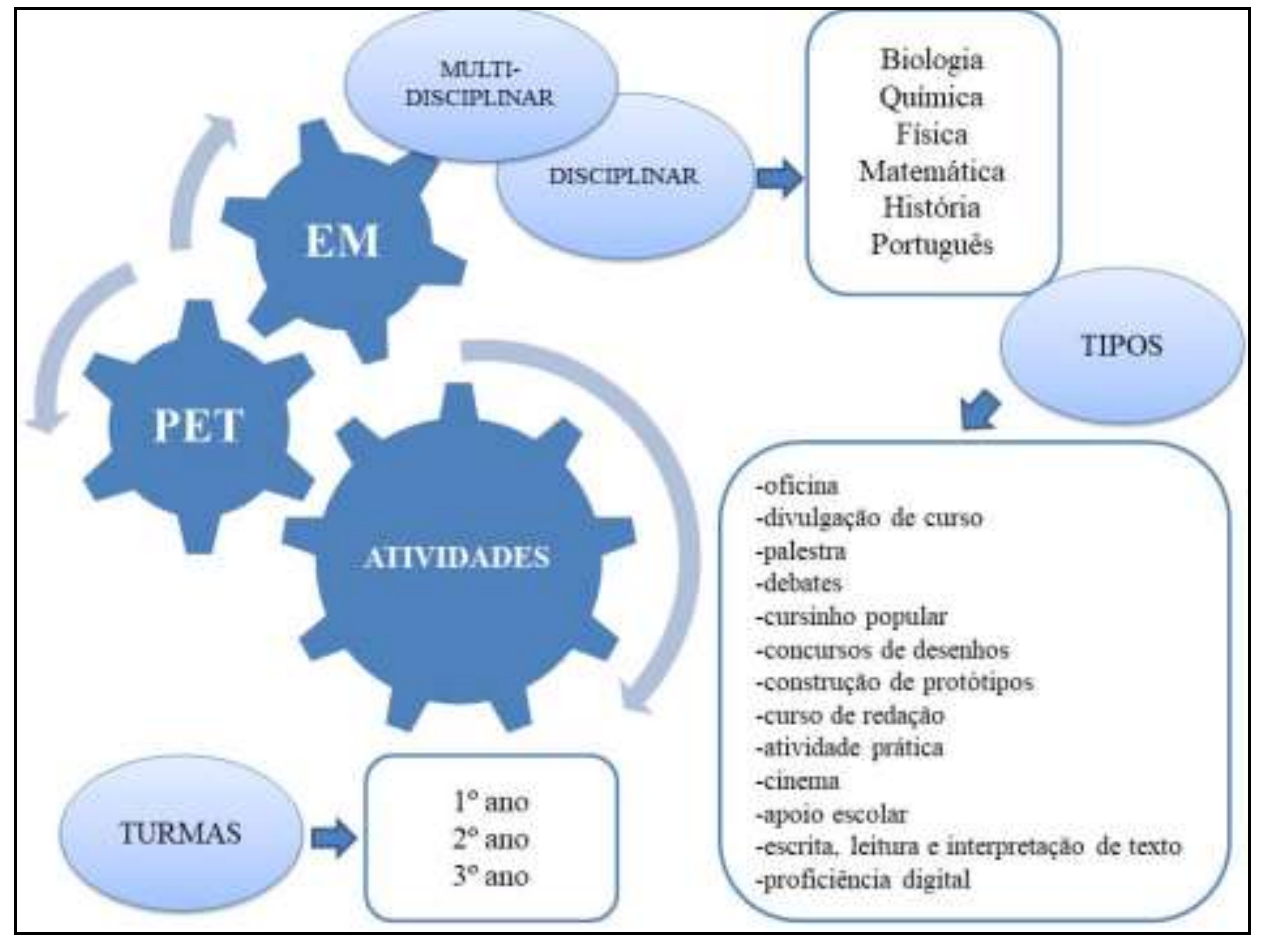

Fonte: Autores.

A Figura 1 apresenta os resultados da classificação das 24 atividades desenvolvidas pelos grupos PET que tinham interação com o Ensino Médio. As disciplinas envolvidas nas atividades foram: Biologia, Química, Física, Matemática, História e Português, com as turmas de $1^{\circ}, 2^{\circ}$ e $3^{\circ}$ ano do Ensino Médio. Entre os tipos de atividades desenvolvidas com as 
turmas de Ensino Médio foi possível identificar a realização de oficina, divulgação de curso, palestra, debates, cursinho popular, concursos de desenhos, competições de carrinhos montados pelos alunos, curso de redação, atividade prática, cinema, apoio escolar, escrita, leitura e interpretação de texto, construção de protótipo de Língua Brasileira de Sinais (LIBRAS) e proficiência digital.

Além das atividades realizadas em sala de aula também ocorreram atividades extraclasse, assim como podem ser encontradas atividades curriculares e complementares. Uma atividade extraclasse é relatada no trabalho de Santos, Pedrosa e Aires (2017) que apresentam o grupo PET-Química da Universidade Federal do Paraná (UFPR) o qual desenvolve atividades de cunho acadêmico, como minicursos, palestras e visitas às Indústrias Químicas, e extensão, com o recebimento de alunos de escolas públicas e particulares no Departamento de Química (DQ) da UFPR. Nessas visitas, os estudantes do ensino básico têm a oportunidade de conhecer a estrutura e funcionamento da Universidade, bem como os laboratórios de pesquisa e ensino presentes no DQ. Primeiramente, são realizados experimentos que abordam conteúdos de Química, como modelos atômicos, pH, tensão superficial e Química Orgânica, de modo a aproximar o que é visto em sala com a prática. Os alunos também são orientados na visita ao departamento, onde visitam salas de aula, laboratórios de ensino e de pesquisa, e nos arredores do campus, apresentando que a Universidade está aberta para recebê-los.

Não foram identificadas atividades específicas nas disciplinas de Geografia, Artes, Educação Física, Sociologia, Filosofia e Inglês. Porém nas atividades multidisciplinares pode-se perceber a relação de Artes com a atividade do concurso de desenhos, por exemplo. Outra atividade multidisciplinar e complementar foi desenvolvida pelo PET-Interdisciplinar Conexões de Saberes com o debate sobre temas como: Juventude e Educação; Ações Afirmativas; Direitos humanos e Acesso Permanência no Ensino Superior Público (Costa, Pimentel \& Rosário, 2016), onde foi possível perceber a relação com a disciplina de Sociologia.

Grande parte das atividades desenvolvidas foi classificada como multidisciplinar, mas também há uma parcela considerada disciplinar, conforme mostrada na figura 1. Entre as atividades multidisciplinares destacamos o trabalho de Freitas, Assis, Socorro e Queiroz (2015) realizado pelo PET- Engenharia Sanitária e Ambiental da Universidade Federal da Bahia, o qual buscou analisar o atual estágio de adoção e implantação dos princípios e dispositivos da Lei 12.056/2011, que trata da Educação Ambiental, nas escolas de ensino médio localizadas na cidade de Salvador, Bahia, por meio das experiências, vivências e práticas do PET. O grupo realizou visitas em escolas estaduais e particulares de ensino médio durante as quais, os integrantes do PET ministraram palestras e realizaram oficinas com o objetivo divulgar conceitos associados à sustentabilidade, por meio, principalmente, da divulgação de ações e práticas de uso racional da água e do descarte correto de pilhas e baterias inservíveis.

Com isso, podemos perceber a variedade das atividades que são realizadas pelos grupos PET e a importância que esses grupos têm para a formação inicial assim como para a formação básica, pois os petianos estão presentes ativamente no ensino básico.

\section{Considerações Finais}

O mapeamento das atividades desenvolvidas pelos grupos PET nos auxiliou compreender melhor os tipos de interações existentes entre esses grupos e o Ensino Médio. O grande número de resultados para os descritores pesquisados incialmente reafirma a consolidação do programa em nível nacional.

O modelo de organização dos grupos PET possibilita diferentes vivências aos petianos e àqueles que participam direta e indiretamente de suas atividades. Também na Educação Básica essas atividades criam espaços para discussões e 
aprendizados que vão além da sala de aula, são momentos de trocas de conhecimentos e que contribuem para o desenvolvimento do pensamento crítico dos alunos.

Foi possível identificar que as atividades desenvolvidas pelos grupos PET que tinham interação com o Ensino Médio, foram desenvolvidas de forma multidisciplinar e disciplinar, estas últimas foram identificadas pertencentes às disciplinas de Biologia, Química, Física, Matemática, História e Português. As turmas participantes pertenciam aos três anos do Ensino Médio $\left(1^{\circ}, 2^{\circ}\right.$ e $\left.3^{\circ}\right)$. E foram identificados diferentes tipos de atividades voltadas à leitura, interpretação, discussão e construção de textos; palestras e debates sobre diferentes temas relevantes; atividades práticas e oficinas nas diferentes áreas de estudo; construções, competições e concursos de materiais elaborados pelos alunos; apoio escolar; cinema; divulgação de cursos de graduação; entre outros cursos específicos ofertados.

Dessa forma, o modelo consolidado pelos grupos PET é uma possibilidade de adoção e parceria de atividades que tenham interação com o Ensino Médio, pensando na melhoria da qualidade do ensino básico, pois essas atividades podem ser associadas aos itinerários formativos previsto na BNCC a partir do novo Ensino Médio; na continuidade dos estudos e na preparação profissional.

Sendo assim, o trabalho realizado poderá ser aprofundado em estudos futuros, a comparação entre o período aqui analisado com o período da pandemia pode ser levado em consideração, assim como expandir as buscas para outras bases de dados ou ainda abordar as atividades realizadas em outros níveis de ensino. Novas pesquisas e novas discussões são necessárias para enriquecer a temática abordada, compartilhando as atividades realizadas pelos grupos PET é possível promover a valorização e possibilitar o alcance às atividades dos grupos.

\section{Referências}

Brasil. (2006). Ministério da Educação. Manual de Orientações Básicas PET. Ministério da Educação. Secretaria de Educação Superior - SESu, 2006. http://portal.mec.gov.br/index.php?option=com_docman\&view=download\&alias=338-manualorientabasicas\&category_slug=pet-programa-de-educacaotutorial\&Itemid=30192

Brasil. (2019). Ministério da Educação. Novo Ensino Médio por Luciano Marques. 2019. http://portal.mec.gov.br/ultimas-noticias/211-218175739/78841-comcarga-horaria-25-maior-aluno-sera-protagonista-na-escolha-da-formacao

Brasil.(2018). Ministério da Educação. Base Nacional Comum $\quad$ Brasília, http://basenacionalcomum.mec.gov.br/images/BNCC_EI_EF_110518_versaofinal_site.pdf

Brasil.(2017). Lei $n^{o} 13.415$, de 16 de fevereiro de 2017. Diário Oficial da União, Brasília, DF, 17 fev. 2017. http://www.planalto.gov.br/ccivil_03/_ato20152018/2017/lei/113415.htm

Bremm, D. (2019). Os filmes e jogos didáticos no trabalho educativo com reeducação alimentar. Revista Insignare Scientia, 2 (3), 92-102. doi: https://doi.org/10.36661/2595-4520.2019v2i3.11186

Ciavatta, M. A. (2018). Reforma do Ensino Médio: uma leitura crítica da lei n. 13.415/2017 - adaptação ou resistência? Holos. 34(4).

Corti, A. P. (2019). Política e significantes vazios: uma análise da reforma do Ensino Médio de 2017. Educação em Revista, Belo Horizonte, 35, 1-20. doi: https://doi.org/10.1590/0102-4698201060

Costa, J., Pimentel, T., \& Rosário, M. J. (2016). PET Interdisciplinar Conexões de Saberes: a troca de conhecimentos na Educação Básica construindo novos caminhos. Revista PET Interdisciplinar e Programa Conexões/UFPA, 1 (1), 31-36. doi: http://dx.doi.org/10.18542/cs.v1i1.3903

Dearo, P. R., Nakayama, J. T. O., \& Rossit, R. A. S. (2017). Potencialidades e fragilidades do Programa de Educação Tutorial: percepções de acadêmicos. Caminho aberto Revista de Extensão do IFSC, 4 (6), 37- 45. doi: http://dx.doi.org/10.35700/ca.2017.ano4n6.p37-45.2071

Fernandes, P. R. (2019). A reforma do Ensino Médio e a produção de desigualdades na educação escolar. Revista Educação, Santa Maria, 44, 1- 19. doi: https://doi.org/10.5902/1984644434731

Ferretti, C. J. (2018). A reforma do Ensino Médio e sua questionável concepção de qualidade da educação. Revista Ensino de Humanidades, 32 (93), 25 - 42. doi: https://doi.org/10.5935/0103-4014.20180028

Freitas, I. S, Assis, M., Socorro, M., \& Queiroz, L. (2015). Educação ambiental nas escolas de ensino médio do estado da Bahia: uma análise a partir das experiências do PET Engenharia Sanitária e Ambiental. Congresso Brasileiro de Engenharia Sanitária e Ambiental, Rio de Janeiro, 28, 1- 4. https://abesnacional.com.br/XP/XP-EasyArtigos/Site/Uploads/Evento29/TrabalhosCompletosPDF/VIII-052.pdf 
Research, Society and Development, v. 10, n. 12, 562101220828, 2021

(CC BY 4.0) | ISSN 2525-3409 | DOI: http://dx.doi.org/10.33448/rsd-v10i12.20828

Gil, A. C. (2018). Como elaborar projetos de pesquisa. $2 . \quad$ reimpr. $6 . \quad$ edição. São Paulo: Atlas, 2018. https://integrada.minhabiblioteca.com.br/\#/books/9788597012934/

Marmitt, D. B. N., Hermel, E. E. S., \& Friedrich, S. P.(2016). Aprendizagem de conteúdos a partir de atividades práticas realizadas em ambientes diferenciados. Revista de Extensão, Santa Maria, 3, 131- 134. http://coral.ufsm.br/revistaccne/index.php/ccnext/article/viewFile/896/644

Martins, L., Espíndola, B. A., Olanda, I. C. C. C.C., \& Nunes, G. P. (2018). Tecendo o texto: oficinas de redação para o ENEM. Anais do 10 Salão Internacional de Ensino, Pesquisa e Extensão - SIEPE Universidade Federal do Pampa, Santana do Livramento, 10 (1), 1- 6. https://periodicos.unipampa.edu.br/index.php/SIEPE/article/view/86602

Pereira, A.N. S. et al. (2018). Metodologia da pesquisa científica. Santa Maria, UFSM, 1, 119. https://repositorio.ufsm.br/bitstream/handle/1/15824/Lic_Computacao_Metodologia-Pesquisa-Cientifica.pdf?sequence=1

Piffero, E. de L., Soares, R. G., Coelho, C. P., \& Roehrs, R. (2020). Metodologias Ativas e o ensino de Biologia: desafios e possibilidades no novo Ensino Médio. Ensino \& Pesquisa, 18 (2), 48- 63. doi: https://doi.org/10.33871/23594381.2020.18.2.48-63

Santos, L. F. F., Pedrosa, L. L., \& Aires, J. A. (2017). Contribuições da Educação Não Formal para Educação Formal: Um Estudo de Visitas de Alunos da Educação Básica ao Departamento de Química da UFPR. ACTIO, Curitiba, 2 (1), 456-473. doi: 10.3895/actio.v2n1.6804

Silva, R. D., Bassani, R., \& Santos, W. C. (2017). Apontamentos sobre a Importância da Construção da Autonomia no Programa de Educação Tutorial. Revista de Graduação da Universidade de São Paulo, 2 (1), 163- 166. doi: https://doi.org/10.11606/issn.2525-376X.v2i1p163-166

Silva, M. M. F., Souza, J. P., Leite, L. B., Morais, B. A., Almeida, C. L., Silva, L. G. F., Miranda, S. V., Costa, M. C. S., \& Araujo, V. H. D. (2017). O PETEducação no contexto da formação acadêmica: as licenciaturas em evidência. Revista on line de Política e Gestão Educacional, 21 (3), 1499-1516. doi: https://doi.org/10.22633/rpge.v21.n3.2017.10593

Silva, M. R. (2018). A BNCC da reforma do Ensino Médio: o resgate de um empoeirado discurso. Educação em Revista, Belo Horizonte, 34, 1-15. doi: https://doi.org/10.1590/0102-4698214130

Souza, R. M., \& Gomes Junior, S. R. (2015). Programa de Educação Tutorial: Avanços na formação em física no Rio Grande do Norte. Revista Brasileira de Ensino de Física, 37 (1), 1-5..doi: https://doi.org/10.1590/S1806-11173711577 